\title{
Erratum: Entanglement entropy of subtracted geometry black holes
}

\author{
Mirjam Cvetič, ${ }^{a, b}$ Zain H. Saleem ${ }^{a}$ and Alejandro Satz ${ }^{a}$ \\ ${ }^{a}$ Department of Physics and Astronomy, University of Pennsylvania, \\ Philadelphia, PA 19104-6396, U.S.A. \\ ${ }^{b}$ Center for Applied Mathematics and Theoretical Physics, University of Maribor, \\ Maribor, Slovenia \\ E-mail: cvetic@physics.upenn.edu, zains@sas.upenn.edu, \\ alesatz@sas.upenn.edu
}

ERratum TO: JHEP09(2014)041

\section{ARXIV EPRINT: 1407.0310}

We compute the entanglement entropy of minimally coupled scalar fields on subtracted geometry black hole backgrounds, focusing on the logarithmic corrections. We notice that matching between the entanglement entropy of original black holes and their subtracted counterparts is only at the order of the area term. The logarithmic correction term is not only different but also, in general, changes sign in the subtracted case. We apply Harrison transformations to the original black holes and find out the choice of the Harrison parameters for which the logarithmic corrections vanish.

In the paper, when evaluating the loop contribution to the entropy given by eq. (3.5), we claim after eq. (3.8) that the curvature scalar $R$ vanishes on our metrics and hence the term $\int_{\Sigma} R$ does not contribute to the entropy. This is mistaken. $R$ vanishes at the horizon in the static case, but not in the rotating case for subtracted geometry. Therefore the results written in (3.19) and (3.21) for the entanglement entropy of rotating subtracted black holes need to be modified. The entanglement entropy for the general four-charge rotating subtracted black holes is given by:

$$
\begin{aligned}
S^{\text {loop }}= & \frac{A_{\Sigma}}{48 \pi \epsilon^{2}}+\frac{1}{72} \frac{a^{2}\left(m+\sqrt{m^{2}-a^{2}}\right)\left(\Pi_{c}-\Pi_{s}\right)^{2}}{m\left(2 m\left(m+\sqrt{m^{2}-a^{2}}\right) \Pi_{c}^{2}+a^{2}\left(\Pi_{s}^{2}-\Pi_{c}^{2}\right)\right)} \log \left(\frac{r_{+}}{\epsilon}\right) \\
& -\frac{1}{180} \frac{m^{3}\left(\Pi_{c}+\Pi_{s}\right)^{3}+\sqrt{\left(m^{2}-a^{2}\right)^{3}\left(\Pi_{c}-\Pi_{s}\right)^{3}}}{m\left[m\left(\Pi_{c}+\Pi_{s}\right)+\sqrt{m^{2}-a^{2}}\left(\Pi_{c}-\Pi_{s}\right)\right]\left[m\left(\Pi_{c}^{2}+\Pi_{s}^{2}\right)+\sqrt{m^{2}-a^{2}}\left(\Pi_{c}^{2}-\Pi_{s}^{2}\right)\right]} \log \left(\frac{r_{+}}{\epsilon}\right),
\end{aligned}
$$


In the case of Kerr-Newman subtracted black hole this reduces to:

$$
\begin{aligned}
S_{\mathrm{KN}-\text { sub }}^{\text {loop }}= & \frac{A_{\Sigma}}{48 \pi \epsilon^{2}}+\frac{1}{72} \frac{a^{2}\left(m+\sqrt{m^{2}-a^{2}}\right)\left(\cosh ^{4} \delta-\sinh ^{4} \delta\right)^{2}}{m\left(2 m\left(m+\sqrt{m^{2}-a^{2}}\right) \cosh ^{8} \delta+a^{2}\left(\sinh ^{8} \delta-\cosh ^{8} \delta\right)\right)} \log \left(\frac{r_{+}}{\epsilon}\right) \\
& -\frac{1}{180} \frac{m^{3}\left(\cosh ^{4} \delta+\sinh ^{4} \delta\right)^{3}+\sqrt{\left(m^{2}-a^{2}\right)^{3}}\left(\cosh ^{4} \delta-\sinh ^{4} \delta\right)^{3}}{m\left(\tilde{\gamma}_{1} \tilde{\gamma}_{2}\right)} \log \left(\frac{r_{+}}{\epsilon}\right),
\end{aligned}
$$

where $\tilde{\gamma_{1}}$ stands for $m\left(\cosh ^{4} \delta+\sinh ^{4} \delta\right)+\left(m^{2}-a^{2}\right)^{1 / 2}\left(\cosh ^{4} \delta-\sinh ^{4} \delta\right)$ and $\tilde{\gamma}_{2}$ stands for $m\left(\cosh ^{8} \delta+\sinh ^{8} \delta\right)+\left(m^{2}-a^{2}\right)^{1 / 2}\left(\cosh ^{8} \delta-\sinh ^{8} \delta\right)$.

Open Access. This article is distributed under the terms of the Creative Commons Attribution License (CC-BY 4.0), which permits any use, distribution and reproduction in any medium, provided the original author(s) and source are credited. 